$\mathrm{E}$ EVALUAR
2019, Vol. 19, No. 3

ISSN $1667-4545$

Recuperado de https://revistas.unc.edu.ar/index.php/revaluar

Laboratorio de Evaluación Psicológica y Educativa Facultad de Psicología - Universidad Nacional de Córdoba

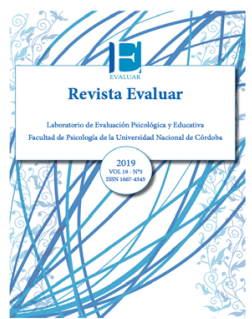

\title{
Evaluación psicométrica de la Escala de Resiliencia para niños mexicanos (GA-RE14)
}

\section{Psychometric Evaluation of the Resilience Scale for Mexican Children (GA-RE14)}

\author{
Norma Ivonne González-Arratia-López-Fuentes ${ }^{1}$ *, \\ Alejandra del Carmen Domínguez-Espinosa ${ }^{2}$, Martha Adelina Torres-Muñoz ${ }^{1}$ \\ 1 - Universidad Autónoma del Estado de México. \\ 2 - Universidad Iberoamericana.
}

Introducción
Estudio 1
Método
Estudio 2
Método
Discusión
Referencias

Recibido: 21/06/2019 Revisado: 20/08/2019 Aceptado: 29/09/2019

\section{Resumen}

Se examinan las propiedades psicométricas de la versión breve de la Escala de Resiliencia para niños de González-Arratia (GA-RE14) con dos estudios. Primero se verifica la estructura tridimensional y consistencia interna con una muestra de 217 escolares (122 niños, 95 niñas) y una Media de edad de 11.14 años $(\mathrm{DE}=.78)$. El Estudio 2 aporta evidencias de convergencia teórica con autoestima y satisfacción con la vida, con 121 participantes ( 59 niños, 62 niñas) con promedio de edad de 10.60 años $(\mathrm{DE}=.67)$. La configuración se comprobó con análisis factorial confirmatorio $(\mathrm{GFI}=.94, \mathrm{AGFI}=.91, \mathrm{RMSEA}=.046 ; \mathrm{NFI}=.79$, $\mathrm{TLI}=.90, \mathrm{CFI}=.92)$. Hay asociación positiva y significativa entre las variables evaluadas, y puntajes más altos en las niñas que en los niños. Esta escala es útil para medición de la resiliencia infantil en contextos académicos y con fines de investigación.

Palabras clave: resiliencia, análisis factorial confirmatorio, consistencia interna, autoestima, satisfacción con la vida

\begin{abstract}
The psychometric properties of the short version of González-Arratia's Resilience Scale for children (GARE14) are examined through two studies. The first one analyzes the three-dimensional structure and internal reliability of a 217 children sample with both genders (122 boys, 95 girls) with an average age of $11.14(\mathrm{SD}=.78)$. Study 2 presents evidence of theoretical convergence with self-esteem and satisfaction with life, with 121 participants (59 boys, 62 girls) aged 9 to 12 years old $(\mathrm{M}=10.60, \mathrm{SD}=.67)$. The configuration is verified by using confirmatory factorial analysis $(\mathrm{GFI}=.94, \mathrm{AGFI}=.91, \mathrm{RMSEA}=.046$; $\mathrm{NFI}=$ $.79, \mathrm{TLI}=.90, \mathrm{CFI}=.92)$. A significant positive association between the evaluated variables, and higher scores in girls than in boys are reported. This scale is useful for the measurement of resilience in children, for academic contexts and for research purposes.
\end{abstract}

Keywords: resilience, confirmatory factorial analysis, internal reliability, self-esteem, satisfaction with life

*Correspondencia a: Norma Ivonne González-Arratia-López-Fuentes. Mariano Matamoros sur 706 Col. Francisco Murguía, Toluca, Estado de México, C.P. 50130. Tel (722) 2721518 Ext 157. E-mail: nigalf@yahoo.com.mx

Cómo citar este artículo: González-Arratia-López-Fuentes, Domínguez-Espinosa \& Torres-Muñoz, Evaluar, 2019, 19(3), 1-19. Recuperado de https:// revistas.unc.edu.ar/index.php/revaluar 


\section{Introducción}

La resiliencia es un concepto dinámico que se refiere a la capacidad de los individuos de afrontar y adaptarse con éxito a una situación adversa. Si bien aún no existe un consenso respecto a su definición (Becoña-Iglesias, 2006; Kalawski \& Haz, 2003), la mayoría de los autores coinciden en referir que implica: 1) la exposición significativa al riesgo y 2) la evidencia de adaptación a pesar de serias amenazas para el desarrollo (Masten, 2007).

Uno de los aspectos más controversiales del estudio de la resiliencia, es su medición. Autores como Pourtois (2014) indican que no es mesurable y otros señalan que "se puede medir a través del riesgo y adaptación positiva" (Luthar \& Bidwell-Zelazo, 2007, p. 514). Pero cuando existen múltiples definiciones e intervienen distintos factores, se dificulta su operacionalización, lo cual tiene importantes implicaciones para su investigación.

La necesidad de contar con instrumentos que ayuden a la comprensión de este constructo ha dado lugar al desarrollo de múltiples instrumentos. Según Ospina-Muñoz (2007, p. 63) "un instrumento con capacidad para abordar el fenómeno resiliente en sus diferentes dimensiones, podría constituir una oportunidad de desarrollo teórico importante en el tema y avanzar en las propuestas de intervención que involucran la promoción de la resiliencia".

El interés creciente por la medición de la resiliencia ha permitido el desarrollo de diversos instrumentos, entre los más utilizados se encuentran: la escala de Connor y Davidson (2003), la escala de Wagnild y Young (1993), la escala de resiliencia para jóvenes de Hurtes y Allen (2001), la escala de autoinforme de resiliencia de Jew, Green y Kroger (1999) y la escala de resiliencia para adolescentes de Oshio, Kaneko, Nagamine y
Nakaya (2003).

Estos instrumentos consideran en su mayoría que la resiliencia es un constructo multidimensional, un ejemplo de ello es la escala de resiliencia de Connor y Davidson (2003), la cual es una de las escalas más utilizadas especialmente en el caso de adultos. Sin embargo, pocos estudios apoyan el modelo factorial de cinco dimensiones de la escala original, pues hay evidencias que indican que se trata más de una estructura de tres factores (tenacidad y autoeficacia, control personal y competencia social; Serrano-Parra et al., 2012). Campbell-Sills y Stein (2007) señalan que dada la inestabilidad de la escala en cuanto a su estructura factorial, se necesitan más estudios que permitan confirmar dicha estructura (Serrano-Parra et al., 2012). La escala de Connor y Davidson (2003) también cuenta con una versión breve de 10 ítems aplicable a muestras de adultos, con adecuada consistencia interna (alfa de Cronbach $=.95)$. Sin embargo, Shin et al. (2018) refieren que aún son necesarios análisis confirmatorios para verificar la validez de la misma.

Lo mismo sucede con la escala de resiliencia de Wagnild y Young (1993) en su versión de 25 ítems, la cual "ha presentado estructuras factoriales inestables y poco claras, según diferentes idiomas y poblaciones" (Sánchez-Teruel \& Robles-Bello, 2015, p. 104) lo que ha llevado a la necesidad de mayor investigación, ahora en su versión de 14 ítems (RS-14; Sánchez-Teruel \& Robles-Bello, 2015).

$\mathrm{Si}$ bien existen varios instrumentos para la medición de la resiliencia con adecuadas propiedades psicométricas, también es importante tomar en cuenta que estos no consideran el entorno social, lo que hace necesario que el instrumento de medida esté diseñado y adaptado a las características específicas de cada cultura (Jowkar, Friborg, \& Hjemdal, 2010). En consecuencia, resulta indispensable construir instrumentos que sean capa- 
ces de evaluar el constructo resiliencia (SalgadoLevano, 2005) tomando en cuenta la población específica a la que está dirigida y el contexto en el cual será utilizado.

La tendencia actual es evaluar constructos a través de técnicas breves o bien con escalas que cuenten con versiones cortas, con la finalidad de indagar de manera rápida el constructo en cuestión, lo que facilita la aplicación en poblaciones infanto-juveniles (Gosling, Rentfrow, \& Swann, 2003; Torreblanca-Murillo, 2017). En el caso de la escala de resiliencia de González-ArratiaLópez-Fuentes y Valdez-Medina (2012), se tiene por objetivo la medición de la resiliencia individual, la cual se conceptualiza como la capacidad que le permite al individuo adaptarse y sobreponerse a las condiciones adversas a las que está expuesto, así como al estrés de la vida cotidiana para enfrentar los retos de manera saludable (Moskovitz, 1983).

Al considerar los antecedentes de la escala de resiliencia, es importante señalar que esta se construyó con base en dos propuestas: 1) los postulados teóricos de Henderson-Grotberg (2006) y 2) el modelo ecológico-transaccional de resiliencia, basado en el modelo ecológico de Bonfenbrenner (1979). Respecto a la primera propuesta, la autora define la resiliencia como "una capacidad humana universal para hacer frente a las adversidades de la vida, superarlas e incluso ser transformado por ellas, e indica que la interacción de factores resilientes proviene de cuatro categorías que son: yo tengo (apoyo externo/soporte social) yo soy y yo estoy (se refiere al desarrollo de la fortaleza psíquica o fuerza interior) y yo puedo (comprende las capacidades interpersonales y de resolución de conflictos)" (GonzálezArratia-López-Fuentes, 2018, p. 20). En cuanto al modelo ecológico, se retomó debido a que se concibe que la resiliencia no depende exclusivamente del individuo, sino también de características del entorno inmediato (como la familia), del grupo social, así como del contexto, los cuales están en constante interacción y relación recíproca, lo que permite a la persona adaptarse a pesar de la adversidad (Rutter, 1999). Así, ambos sirvieron de base para la elaboración de los ítems de la escala que aquí se presenta.

Con la escala original en su versión de 32 ítems se han realizado múltiples estudios con análisis factorial exploratorio y confirmatorio, en los que se han informado tres dimensiones. La primera dimensión es el factor protector interno (FPI), el cual mide habilidades para la solución de problemas. La segunda dimensión corresponde al factor protector externo (FPE), el cual evalúa la percepción que tiene el individuo sobre la posibilidad de contar con apoyo de la familia que promueve la resiliencia. La tercera dimensión es el factor empatía (FE) que mide el comportamiento altruista y prosocial. También se han identificado estudios en los que ha sido aplicada la escala de González-Arratia-López-Fuentes y ValdezMedina (2012) en población normal en diferentes etapas del desarrollo, desde niños hasta adultos mayores. Por ejemplo en México, Plata (2013) en su estudio con niños confirma que se trata de una escala tridimensional, al igual que el estudio de Toribio-Perez (2017) con muestras de adolescentes, e incluso se ha informado del uso de la escala con adultos (Castro, 2016; Rocha-Romero, 2017) y adultos mayores (Díaz, 2019). Este grupo de estudios ha reportado adecuadas propiedades psicométricas para la escala, lo que lleva a considerar que es uno de los instrumentos más utilizados en México para la medición de la resiliencia.

En cuanto a la utilidad de la escala de González-Arratia-Lopez-Fuentes y ValdezMedina (2012), esta ha sido aplicada en muestras de niños en contextos de vulnerabilidad psicosocial con adecuados resultados psicométricos (González-Arratia-López-Fuentes, Morelato, 
González-Escobar, \& Ruíz-Martínez, 2018). A la fecha también existen datos sobre su validez en relación con otras escalas de resiliencia para niños: González-Arratia-López-Fuentes y ValdezMedina (2012, p. 680) obtuvieron "valores de correlación positivos significativos con la escala de Hurtes y Allen (2001) y con la de Jew et al. (1999; $r_{(405)}=.55, p<.001 ; r_{(405)}=.58, p<.001$ respectivamente) lo que proporciona evidencia de que están midiendo el mismo constructo".

En el estudio más reciente de GonzálezArratia-López-Fuentes (2018), se obtuvo un instrumento con tres dimensiones y 13 ítems. Si bien se obtuvo un buen ajuste con este modelo, hay que tener en cuenta cierta limitación, dado que el factor empatía (FE) quedó conformado solo por dos ítems. Por ese motivo en el presente estudio se prefirió comprobar la versión, ahora con 14 ítems, ya que existe un amplio acuerdo en cuanto a un mínimo deseable de 3 indicadores por factor para que el factor sea admisible. De ahí que se aplicó la condición de que cada factor tenga asociadas por lo menos tres variables observadas (Hair, Anderson, Tatham, \& Black, 2004). Además de este criterio estadístico para la inclusión de un indicador, específicamente el ítem R7FE (Yo soy acomedido y cooperador) está teóricamente asociado al factor que le corresponde, por lo que se lo incluye siguiendo la recomendación de OrgazBaz (2008, p. 26): "si el interés teórico es suficiente debería mantenerse en el modelo".

A pesar de que se cuenta con una escala viable para la medición de la resiliencia con propiedades psicométricas satisfactorias, para facilitar su uso, sobre todo en casos en donde se requiere la exploración de otras escalas, se considera importante contar con una versión breve de la misma, que sea más sencilla de utilizar. Adicionalmente, resulta relevante tomar en cuenta que en general, las pruebas psicológicas requieren de una constante revisión conceptual de los ítems, debido a "la posible pérdida de vigencia de alguno de estos" (Hernández-Contreras \& Rivera-Ottenberger, 2018, p. 145), y sobre todo porque "el proceso de validación no termina, es permanente y exige comprobaciones empíricas continuas" (MonteroRojas, 2013, p. 118).

Debido a que es aconsejable revisar el contenido de los instrumentos ya existentes (PerpiñáGalvañ, Richart-Martínez, Cabañero-Martínez, \& Martínez-Durá, 2011), y a que las formas cortas de una escala se pueden utilizar con tanto éxito como la versión original (Loo \& Thorpe, 2000; Sârbescu, Rusu, \& Costea, 2012), en esta investigación se emplea la versión breve conformada por 14 ítems, la cual se ha denominado GA-RE14. Estos ítems corresponden al estudio más reciente del instrumento (González-ArratiaLópez-Fuentes, 2018) más la incorporación del ítem R7FE que, como se mencionó anteriormente, sirve al propósito de confirmar el ajuste del modelo tridimensional sin perder su interpretabilidad para su uso en otras muestras.

En lo concerniente a las diferencias entre hombres y mujeres, la información al respecto es contradictoria, ya que se han informado evidencias de que las mujeres son más resilientes (González-Arratia-López-Fuentes \& ValdezMedina, 2012) mientras que otros autores han informado que los hombres son más resilientes (Stratta et al., 2013), e incluso que no hay diferencia alguna (Kenneally-Nicholas, 1993; PradoÁlvarez \& del Águila-Chávez, 2003). Esto lleva a la necesidad de continuar analizando las posibles diferencias y/o similitudes entre niños y niñas.

Adicionalmente, es importante tomar en cuenta otras cuestiones relativas a la validez. Dado que se recomienda sumar evidencias de validez de la escala en distintas muestras, estudiamos la relación de la escala con dos importantes variables. Una de ellas es la autoestima, debido a que las personas con una evaluación favorable de 
sí mismas muestran un afrontamiento positivo de los problemas, e incluso se ha demostrado que es una importante variable que predice la resiliencia (Balgiu, 2017; Benetti \& Kambouropoulos, 2006). Del mismo modo, se estudia la relación con la variable satisfacción con la vida, que refiere a la evaluación global que realizan las personas acerca de su propia vida, debido a los beneficios que ha mostrado sobre el funcionamiento y ajuste psicológico de los individuos (Ruíz-González, Medina-Mesa, Zayas, \& Gómez-Molinero, 2018). Con respecto a esta variable, se ha demostrado que los jóvenes que puntúan como satisfechos y ligeramente satisfechos sobre sus condiciones de vida muestran mayor capacidad resiliente (BernalRomero, Daza-Pinzón, \& Jaramillo-Acosta, 2015; Casazola-Larota \& Vargas-Mamani, 2018). Además, numerosos estudios indican que tanto la autoestima como la satisfacción con la vida son importantes variables que permiten un resultado positivo frente al estrés (Rodríguez-Naranjo \& Caño-González, 2012), en este caso, el hecho de vivir en situación de pobreza (Palomar-Lever, 2015).

Así, en la presente investigación se llevan a cabo dos estudios. El primero de ellos tiene como objetivos: 1) verificar la estructura tridimensional de la escala breve (GA-RE14) con análisis factorial confirmatorio (AFC) y 2) evaluar la fiabilidad de la escala. El segundo estudio en una segunda muestra tiene como objetivos: 1) examinar la validez de constructo a partir de fuentes externas de la escala, al evaluar su relación con las variables autoestima y satisfacción con la vida; 2) comparar el nivel de resiliencia, autoestima y satisfacción con la vida entre niños y niñas.

De acuerdo con los objetivos, se espera que: la versión breve (GA-RE14) de 14 ítems, conste de tres dimensiones consistentes y que exista una fiabilidad aceptable según el criterio de Oviedo y Campo-Arias (2005). Asimismo, se tiene como expectativa encontrar correlaciones positivas con autoestima y con satisfacción con la vida, y se espera, según el criterio de Lévy-Mangin y VarelaMallou (2006), una correlación entre los constructos no mayor a .5. Del mismo modo, se tiene como expectativa que existan diferencias entre niños y niñas en los tres constructos evaluados.

A la fecha, en México no se cuenta con escalas para la medición de la resiliencia infantil, por lo que el hecho de contar con un instrumento breve para la evaluación de este importante constructo resulta relevante sobre todo en el contexto educativo, lo que justifica su estudio con el sentido de facilitar su medición y así obtener evidencia empírica que sirva de base para el diseño de programas de intervención en niños de países hispanoparlantes.

\section{Estudio 1. Comprobar la estructura tridimensional de la escala de resiliencia (GA- RE14) con 14 ítems \\ Método}

Participantes

Se realizó un estudio transversal con un diseño de tipo instrumental (Montero \& León, 2002). Con un muestreo no probabilístico de tipo intencional, la Muestra 1 quedó constituida por 217 participantes, de los cuales el 56.2\% son niños $(n=122)$ y el $43.8 \%$ niñas $(n=95)$ con un rango de edad de 9 a 13 años $(\mathrm{M}=11.14, \mathrm{DE}=$ .78). Los participantes de este estudio pertenecen a tres escuelas públicas consideradas de alta vulnerabilidad. Los criterios de inclusión fueron: ser alumnos de educación básica de $4^{\circ}, 5^{\circ}$ y $6^{\circ}$ grado, inscritos en escuelas públicas pertenecientes a San Pablo Autopan, que es una localidad que pertenece al Municipio de Toluca, Estado de México. Dicha localidad es considerada el municipio en el cual se concentra el mayor número de personas en 
situación de pobreza, según el Consejo Nacional de Evaluación de la Política de Desarrollo Social (CONEVAL, 2013) que es la institución gubernamental que evalúa los índices de pobreza en México. Es importante señalar que el CONEVAL (2013) define como persona en situación de pobreza a aquella que presenta al menos una carencia social (de los seis indicadores de pobreza) y no tiene un ingreso suficiente para satisfacer sus necesidades alimentarias y no alimentarias. Los indicadores que se consideran corresponden a carencias por: 1) rezago educativo, 2) acceso a los servicios de salud, 3) acceso a la seguridad social, 4) calidad y espacios de vivienda, 5) servicios básicos de vivienda y 6) acceso a la alimentación (que representa el $41.8 \%$ de personas pobres). Específicamente en esta localidad donde se realizó el estudio, se ha reportado que el $6.86 \%$ de la población está en situación de pobreza extrema (CONEVAL, 2008-2018).

\section{Instrumentos}

Se elaboró una ficha de datos sociodemográficos que incluye información sobre: edad, sexo, promedio de calificaciones y escuela de procedencia. La evaluación de la resiliencia se hizo con la Escala de Resiliencia GA-RE14, la cual tiene el objetivo de evaluar la capacidad de la persona de adaptarse y ajustarse "después de haber estado sometida a situaciones de adversidad" (LópezFuentes \& Calvete, 2016, p. 111). Se trata de una escala tipo Likert en su versión breve de 14 ítems, en la que se le solicita al participante que al momento de responder tenga presente una situación que considere de crisis o en la que haya tenido algún problema, pensando en qué medida cree que se sintió o se comportó en esa situación, con cinco opciones de respuesta, que son: 1 (Nunca) 2 (Pocas veces) 3 (Algunas veces) 4 (Muchas ve- ces) y 5 (Siempre).

En el estudio previo de González-ArratiaLópez-Fuentes (2018) se obtuvieron 3 factores: el factor protector interno (FPI) con 8 ítems (R17, R18, R20 R21, R25, R26, R27, R30), factor protector externo (FPE) con 3 ítems (R14, R15, R16) y el factor empatía (FE) con 3 ítems (R7, R8, R9) con una varianza total explicada de $44.63 \%$ y una confiabilidad de alfa de Cronbach total de $\alpha=$ .860 .

\section{Procedimiento}

Se contó con la autorización de la institución, así como con el consentimiento informado de los padres, y el consentimiento de los participantes; las aplicaciones de la escala se llevaron a cabo de manera voluntaria y anónima en las respectivas aulas y horarios académicos. Se explicó el objetivo de la investigación y las investigadoras atendieron las dudas que surgieron en el momento de la aplicación. Todos los participantes completaron la escala en un tiempo aproximado de 20 minutos. El protocolo del presente estudio forma parte del proyecto de investigación con clave 4645/2019SF y ha sido avalado por el Comité de Ética de la U. A. E. Mex. (Clave: 2019/05), el estudio se realizó bajo los estándares éticos que indica la Asociación Americana de Psicología (2010).

\section{Análisis de Datos}

Se realizó un análisis descriptivo y se evaluó el supuesto de normalidad univariante con el contraste de Kolmogorov-Smirnov y Shapiro-Wilk, así como el de normalidad multivariante que propone Mardia (1970, como se citó en GonzálezÁlvarez, Abad-González, \& Lévy-Mangin, 2006) 
para contrastar "si la asimetría y la curtosis multivariantes del conjunto de variables observables permiten asumir o no la hipótesis de normalidad" (González-Álvarez et al., 2006, p. 34). Se utilizó un análisis de correlación para verificar la discriminación de los ítems.

El AFC se llevó a cabo con el método de estimación de máxima verosimilitud (ML) para probar la estructura hipotetizada (Lloret-Segura, Ferreres-Traver, Hernández-Baeza, \& TomásMarco, 2014). De acuerdo con las recomendaciones de $\mathrm{Hu}$ y Bentler (1998) se consideraron los índices de ajuste absoluto (Chi-cuadrado y $\chi^{2} / g l$, GFI, AGFI $\geq .90$ y RMSEA y PCLOSE), así como los índices de ajuste incremental (CFI y NFI) e índices de ajuste de parsimonia (PNFI, PGFI $\geq$ .90; Verdugo, Crespo, Badía, \& Arias, 2008). En general, se recomienda que los valores del CFI y NNFI sean $\geq .90$, lo que indica un buen ajuste para modelos confirmatorios, en el caso de los valores del RMSEA, valores $\leq .06$ indican un excelente ajuste y valores que van de .06 a .08 indican un ajuste moderado del modelo. Se realizó el cálculo de confiabilidad alfa de Cronbach $(\alpha)$, y según el criterio de Oviedo y Campo-Arias (2005) se consideraron los valores de alfa entre .80 y .90 como los más recomendables. Los datos se analizaron con el programa IBM SPSS (IBM Corporation, 2011) y Amos versión 20 (Arbuckle, 2011).

\section{Resultados}

En la Tabla 1 se muestran los datos descriptivos de la primera muestra, en la que se observa que todos los ítems presentan puntuaciones promedio por arriba del valor medio que es 3 . El ítem R19FPI (Soy firme en mis decisiones) presentó la media más baja, mientras que el ítem R15FPE (Tengo deseos de triunfar) obtuvo la media más alta. Respecto a la normalidad univariada, se ob- tuvo que los ítems en general tienden a una distribución leptocúrtica, y la prueba KolmogorovSmirnov indica una distribución no normal de los ítems. De acuerdo con Ferrando y AnguianoCarrasco (2010), se observa que tanto la asimetría como curtosis están por sobre +/- 1.5 en los ítems: R14FPE, R15FPE, R16FPE y R30PFI. También fue necesario evaluar la hipótesis de normalidad multivariada de Mardia (1970, en GonzálezÁlvarez et al., 2006), la cual resultó de 58.45, lo cual permite asumir que de manera conjunta los ítems presentan "una curtosis significativamente distinta de la de una norma multivariante" (González-Álvarez et al., 2006, p. 42).

Adicionalmente, se llevó a cabo el cálculo de discriminación con las puntuaciones de cada uno de los ítems y el puntaje total de la escala, mediante el coeficiente de correlación Rho de Spearman, el que mostró que los valores de la correlación son positivos y fluctúan entre .25 a .54 . Según el criterio de Mondragón-Barrera (2014), estos valores indican que existe una asociación de media a considerable, por lo que se conservan todos los ítems (ver Tabla 1).

\section{Análisis factorial confirmatorio}

Se comenzó por especificar el modelo definido por tres variables latentes y 14 indicadores; la estimación se realizó mediante el método de ML. No se obtuvo normalidad multivariante como ya se mencionó. No obstante, se continuó con el análisis y se tomaron en cuenta las recomendaciones de Hair et al. (2004) con respecto al tamaño muestral, que indican que para el método ML se use un rango de entre 100 y 200 observaciones. En este caso, la muestra es superior a este criterio, por lo que se procedió al análisis. Respecto a la bondad de ajuste del modelo, se obtuvieron los siguientes índices: $\mathrm{X}_{(74)}^{2}=1.62, p<.001$. Sin em- 
Tabla 1

Datos descriptivos Escala de Resiliencia 14 ítems.

\begin{tabular}{|c|c|c|c|c|c|c|c|c|}
\hline $\begin{array}{l}\mathrm{N}^{\circ} \text { de Ítem } \\
\text { versión } \\
\text { original }\end{array}$ & $\begin{array}{c}\mathrm{N}^{\circ} \text { de ítem } \\
\text { nueva } \\
\text { versión }\end{array}$ & & Min-Max & $\mathbf{M}$ & DE & Asimetría & Curtosis & rho \\
\hline R7FE & R1FE & $\begin{array}{l}\text { Yo soy acomedido y } \\
\text { cooperador }\end{array}$ & $1-5$ & 3.8 & 0.93 & -0.83 & 0.34 & .46 \\
\hline R8FE & $\mathrm{R} 2 \mathrm{FE}$ & Yo soy amable & $2-5$ & 3.9 & 0.93 & -0.88 & 0.04 & .51 \\
\hline R9FE & R3FE & Soy compartido & $1-5$ & 4.0 & 0.86 & -0.90 & 0.60 & .42 \\
\hline R14FPE & R4FPE & $\begin{array}{l}\text { Tengo personas que me quieren } \\
\text { a pesar de lo que sea o haga }\end{array}$ & $1-5$ & 4.4 & 0.84 & -1.86 & 3.34 & .28 \\
\hline R15FPE & R5FPE & Tengo deseos de triunfar & $2-5$ & 4.7 & 0.52 & -2.48 & 7.22 & .25 \\
\hline R16FPE & R6FPE & Tengo metas a futuro & $1-5$ & 4.6 & 0.75 & -2.20 & 4.82 & .39 \\
\hline R17FPI & R7FPI & $\begin{array}{l}\text { Estoy dispuesto a } \\
\text { responsabilizarme de mis actos }\end{array}$ & $2-5$ & 4.2 & 0.79 & -1.15 & 1.18 & .40 \\
\hline R18FPI & R8FPI & $\begin{array}{l}\text { Estoy siempre pensando } \\
\text { la forma de solucionar mis } \\
\text { problemas }\end{array}$ & $1-5$ & 4.0 & 0.87 & -1.05 & 0.92 & .42 \\
\hline R19FPI & R9FPI & Soy firme en mis decisiones & $1-5$ & 3.6 & 0.99 & -0.44 & -0.61 & .54 \\
\hline R21FPI & R10FPI & $\begin{array}{l}\text { Me siento preparado para } \\
\text { resolver mis problemas }\end{array}$ & $1-5$ & 3.8 & 0.93 & -0.57 & -0.31 & .53 \\
\hline R25FPI & R11FPI & $\begin{array}{l}\text { Puedo buscar la manera de } \\
\text { resolver mis problemas }\end{array}$ & $1-5$ & 3.9 & 0.99 & -0.93 & 0.12 & .48 \\
\hline R26FPI & R12FPI & $\begin{array}{l}\text { Puedo imaginar las } \\
\text { consecuencias de mis actos }\end{array}$ & $1-5$ & 4.1 & 0.98 & -1.11 & 0.36 & .46 \\
\hline R27FPI & R13FPI & $\begin{array}{l}\text { Puedo reconocer lo bueno y lo } \\
\text { malo para mi vida }\end{array}$ & $1-5$ & 4.1 & 0.96 & -1.18 & 0.79 & .41 \\
\hline \multirow[t]{2}{*}{ R30FPI } & R14FPI & Puedo aprender de mis errores & $1-5$ & 4.3 & 0.92 & -1.70 & 2.55 & .46 \\
\hline & $\begin{array}{l}\text { Puntaje } \\
\text { Total }\end{array}$ & & $33-67$ & 58.32 & 6.28 & -1.5 & 2.60 & 1 \\
\hline
\end{tabular}

bargo, y en virtud de que chi-cuadrado es sensible al tamaño de la muestra, también se consideraron otros índices de ajuste absolutos (GFI $=.93$, AGFI $=.90$, RMSEA $=.05)$ comparativos (NFI $=.76, \mathrm{TLI}=.86, \mathrm{CFI}=.89)$ e índices de ajuste de parsimonia $(\mathrm{PNFI}=.62$, PGFI $=.65$, PCFI $=$ .72). Debido a que los valores de los índices obtenidos (absolutos, comparativos, de parsimonia) resultaron inferiores a lo esperado, indicando un ajuste inadecuado a los datos, se consideraron los índices de modificación (IM). Para este propósi- to se comprobó un modelo reespecificado. Dada la relación entre los ítems R21FPF y R25FPI, se observó que requerían ser reespecificados, lo que permitió tener un modelo adecuado en el que se obtuvo que $\mathrm{X}^{2}{ }_{(73)}=1.45, p<.006$. Los índices de ajuste absolutos fueron superiores a .90 (GFI $=.939, \mathrm{AGFI}=.912)$. El índice RMSEA mostró un excelente ajuste (.046). Respecto a los índices de ajuste incremental, uno de tres índices mostró valores por debajo a $.85(\mathrm{NFI}=.79$, TLI $=$ .903 , CFI $=.92)$. Cinco de estos seis índices in- 
dican un buen ajuste del modelo, lo cual indica una ligera mejora, y que el modelo se adapta a los criterios señalados por Abad, Garrido, Olea y Ponsoda (2006), de modo que se decidió adoptar este modelo.

Debido a que los datos no cumplen con el supuesto de normalidad multivariada, la estimación de máxima verosimilitud se llevó a cabo con el procedimiento bootstrapping propuesto por Bollen y Stine (1990). En el caso de este estudio, la estimación se realizó con 200 remuestreos para obtener un mejor ajuste (IC al 90\%). Los resultados para la escala en cuanto a errores estándar oscilaron entre .003 y .013 ( $p=.132$; Abad, Olea,

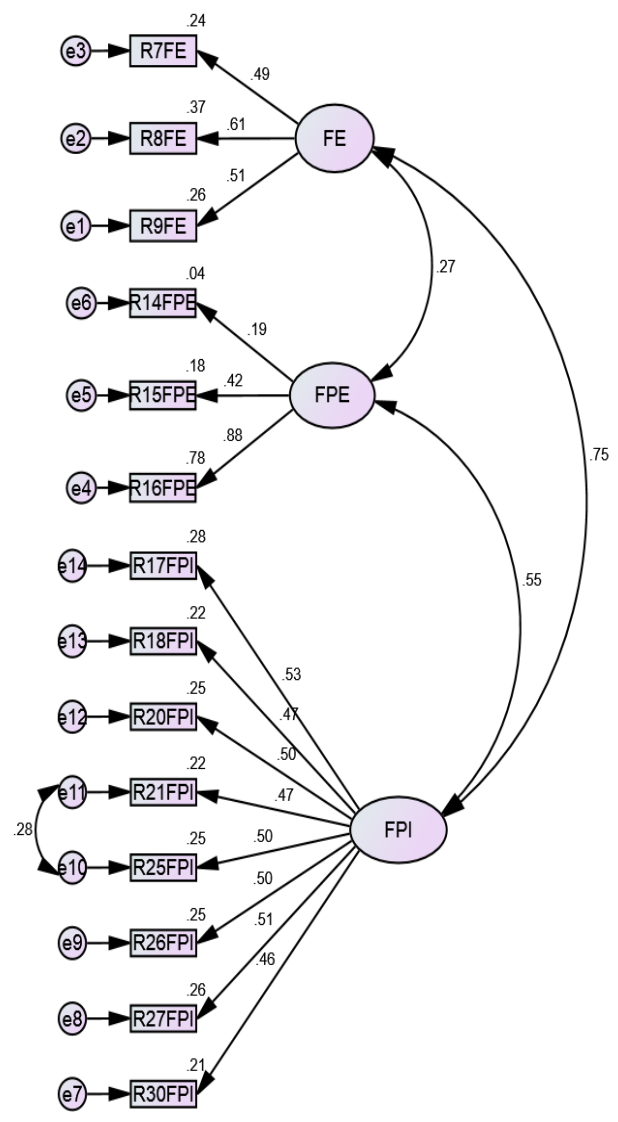

\section{Figura 1}

Modelo final estandarizado para la Escala de Resiliencia.
Ponsoda, \& García, 2011; Byrne, 2010; Hancock \& Nevitt, 1999; Fouladi, 1998).

También se consideró pertinente verificar la consistencia interna en la medición del constructo. Se recomienda que este índice sea superior a .70. En el caso del FPI, la confiabilidad compuesta resultó ser adecuada $(\mathrm{CR}=.719$; Hair et al., 2004) y, respecto a los factores FPE y FE, si bien exceden el umbral de .50, no alcanzaron el criterio recomendado (Rial, Varela-Mallou, Abalo, \& Lévy-Mangin, 2006). En la Figura 1, se muestra la solución final estandarizada del modelo de tres factores de la escala breve de resiliencia.

\section{Confiabilidad de la escala}

La consistencia interna de la escala fue evaluada por medio del cálculo alfa de Cronbach, el cual fue $\alpha=.775$ para la escala total. Por dimensión, los resultados mostraron que, en el caso del factor FPI, fue $\alpha=.726$, para el factor FPE $\alpha=$ .419 y para el factor FE $\alpha=.546$.

\section{Estudio 2. Evidencias de Convergencia teórica de la Escala de Resiliencia con las variables autoestima $y$ satisfacción con la vida $y$ diferencias según la variable atributiva sexo Método}

Participantes

Se evaluó a 121 participantes, quienes conformaron la segunda muestra, de los cuales 59 son niños (48.8\%) y 62 niñas $(51.2 \%)$ de 9 a 12 años $(\mathrm{M}=10.6, \mathrm{DE}=.67)$. Todos son estudiantes que pertenecen a dos instituciones de educación básica, ubicadas en la localidad de San Felipe del Progreso del Municipio de Toluca, que es considerada como uno de los municipios con mayor porcentaje de población en situación de pobreza 
extrema $(43.4 \%)$ y carencia por acceso a la alimentación (CONEVAL, 2013) de acuerdo con el informe de situación de pobreza y rezago social descrito anteriormente.

\section{Instrumentos}

Se aplicó una ficha de datos sociodemográficos como la que se indicó en el Estudio 1. La resiliencia fue evaluada con la escala GA-RE14 como se explicó anteriormente. En este segundo estudio, se obtuvo un coeficiente alfa de Cronbach total de $\alpha=.845$. Adicionalmente, se incluyeron las escalas de autoestima y satisfacción con la vida. Respecto a la escala de autoestima, consta de 25 ítems y 4 opciones de respuesta ( 4 = Siempre; 3 = Muchas veces; 2 = Pocas veces; $1=$ Nunca). Está dividida en seis dimensiones: yo, familia, fracaso, trabajo intelectual, éxito y afectivo-emocional. En el estudio previo de González-ArratiaLópez-Fuentes (2018), se indica que en conjunto explican el $44.08 \%$ de la varianza total, con un alfa de Cronbach de .724 y una confiabilidad compuesta de .703 .

La variable satisfacción con la vida fue evaluada con la escala de Diener, Emmons, Larsen y Griffin (1985), que consta de 5 ítems y 7 opciones de respuesta que van de 1 (Estoy muy en desacuerdo) hasta 7 (Estoy muy de acuerdo) $\mathrm{y}$ que mide el juicio global que hacen las personas sobre su vida. Ha sido validada en México por Padrós-Blázquez, Gutiérrez-Hernández y Medina-Calvillo (2015), quienes informaron un modelo unifactorial con una confiabilidad alfa de Cronbach de .832 .

\section{Procedimiento}

Se solicitó la autorización a la institución educativa, así como el consentimiento a los padres y/o tutores y el consentimiento informado a los participantes. Todos fueron informados del objetivo de la investigación, y la participación fue voluntaria y anónima. La aplicación de las 3 escalas se realizó de manera colectiva en las respectivas aulas y en horarios académicos en un tiempo aproximado de 30 minutos.

\section{Análisis de datos}

Debido a que no se cumple el supuesto de normalidad, se utilizó el cálculo de Rho de Spearman, para determinar la validez convergente del constructo resiliencia con las variables autoestima y satisfacción con la vida. La prueba $t$ de Student se utilizó para analizar las posibles diferencias entre niños y niñas. Los análisis se hicieron con el programa SPSS versión 20 (IBM Corporation, 2011).

\section{Resultados}

Mediante el análisis de correlación Rho de Spearman se encontró que las correlaciones obtenidas entre el puntaje total de la escala de resiliencia y el de la variable autoestima indican una asociación positiva y significativa, tal como se esperaba $(r=.36, p<.001)$. De igual forma, el puntaje total de resiliencia correlacionó de manera positiva y significativa con las puntuaciones de la variable satisfacción con la vida $(r=.35$, $p<.001)$. De manera específica, los resultados mostraron que FE tenía una baja pero significativa correlación con el puntaje total de autoestima $(r=.29, p<.001)$, y FPI obtuvo un valor de correlación bajo con satisfacción con la vida ( $r$ $=.31, p<.001$ ). Respecto a FPE, no se encontró asociación significativa con satisfacción con la 
vida (ver Tabla 2).

Tabla 2

Medias, desviaciones estándar y correlaciones de las escalas de resiliencia, autoestima y satisfacción con la vida.

\begin{tabular}{rccccccc}
\hline & Resiliencia & FPI & FPE & FE & Min-Max & $\boldsymbol{M}$ & $\boldsymbol{D E}$ \\
\hline Autoestima & $.36^{* *}$ & $.29^{* *}$ & $.33^{* *}$ & $.29^{* *}$ & $45-72$ & 61.38 & 7.72 \\
Satisfacción con la Vida & $.35^{* *}$ & $.31^{* *}$ & .03 & $.34^{* *}$ & $15-35$ & 27.88 & 5.21
\end{tabular}

Nota. (FPI) Factor protector interno, (FPE) Factor protector externo, (FE) Factor empatía. ${ }^{* *}$ ) las correlaciones son significativas a $p<.001$ (bilateral).

Respecto al sexo, hubo diferencias entre niños y niñas únicamente en FPE, en el que se observó que las niñas obtuvieron promedios ligeramente más altos que los niños. En el resto de las variables no se hallaron diferencias significativas entre niños y niñas (ver Tabla 3).

Tabla 3

Diferencias de resiliencia, autoestima y satisfacción con la vida entre niños y niñas.

\begin{tabular}{|c|c|c|c|c|c|}
\hline & \multicolumn{2}{|c|}{$\begin{array}{l}\text { Niños } \\
\mathrm{n}=59\end{array}$} & \multicolumn{2}{|c|}{$\begin{array}{l}\text { Niñas } \\
\mathrm{n}=62\end{array}$} & \multirow{2}{*}{$\begin{array}{c}t \\
\boldsymbol{t}_{(119)}\end{array}$} \\
\hline & $M$ & $D E$ & $M$ & $D E$ & \\
\hline 1. FPI & 4.34 & 0.47 & 4.39 & 0.54 & -0.53 \\
\hline 2. FPE & 4.71 & 0.31 & 4.83 & 0.30 & $-2.13 * *$ \\
\hline 3. FE & 4.16 & 0.48 & 4.31 & 0.66 & -1.41 \\
\hline $\begin{array}{l}\text { 4. Puntaje } \\
\text { total Resi- } \\
\text { liencia }\end{array}$ & 61.42 & 5.17 & 62.63 & 6.53 & $1-12$ \\
\hline $\begin{array}{l}\text { 5. Autoes- } \\
\text { tima }\end{array}$ & 60.05 & 7.90 & 62.66 & 7.37 & -1.87 \\
\hline $\begin{array}{l}\text { 6. Satisfac- } \\
\text { ción con la } \\
\text { Vida }\end{array}$ & 27.93 & 5.45 & 27.83 & 5.02 & 0.09 \\
\hline
\end{tabular}

Nota. $* * p<.05$.

\section{Discusión}

El objetivo de este estudio fue analizar las propiedades psicométricas de la versión breve de la escala de resiliencia denominada GA-RE14.
Para ello, se llevaron a cabo dos estudios. El primero de ellos permitió comprobar empíricamente la dimensionalidad de la escala en su versión breve. El modelo se sometió a prueba, considerando diferentes índices.

Los resultados mostraron que el modelo tridimensional hipotetizado presentaba un índice $\chi^{2}$ significativo para el modelo propuesto. El resto de los índices obtenidos (de ajuste absoluto, incremental, y de parsimonia) resultaron adecuados en su mayoría, lo que sugiere que el modelo teórico se ajusta parcialmente a los datos empíricos. Esta evidencia indica que esta nueva versión de la escala de resiliencia es apropiada para la medición del constructo, lo que adicionalmente brinda soporte a la noción de la multidimensionalidad de la resiliencia (Hurtes \& Allen, 2001). Sin embargo, es importante señalar que en este estudio se dio el caso de que algunos índices ajustan mejor a los datos que otros (Verdugo et al., 2008), y que, en términos estadísticos, puede deberse a que los datos obtenidos dependen de la muestra analizada (Byrne, 2010). Si bien fue necesario hacer una reespecificación del modelo, esto ayudó a mejorar el ajuste, sin perder de vista su base conceptual. Esta es una estrategia que suele utilizarse con la finalidad de asegurar ciertas garantías de aceptabilidad (Lévy-Mangin, Martín-Fuentes, \& Román-González, 2006).

Por otro lado, es importante señalar que el hecho de que los ítems resultaron ser variables no normales no impide el uso de métodos de es- 
timación tradicionales tales como la estimación de máxima verosimilitud (Lévy-Mangin et al., 2006). Por lo tanto, se optó por las técnicas como bootstrapping, la cual permitió subsanar esta situación, y evitar afectar el análisis por la falta de normalidad, tal y como lo sugiere Byrne (2010). Es decir, se utilizó un método de estimación más robusto para obtener estimaciones apropiadas, atendiendo al hecho de que en ciencias sociales no siempre es posible obtener observaciones que cumplan con el supuesto de normalidad (OrdazBaz, 2008). Así, sobre la base de estos hallazgos, se puede considerar que el modelo propuesto se ajusta de manera aceptable a los datos, lo que representa una aproximación y una posibilidad para la medición del constructo resiliencia.

De esta forma, en el presente estudio, se puso a prueba la estructura tridimensional de la escala GA-RE14, con resultados que aportan evidencia de la adecuación de este modelo y confirman sus dimensiones, así como la correspondencia de los ítems con cada factor. Es decir, los 14 ítems son congruentes con el factor que les corresponde al igual que en estudios previos (González-ArratiaLópez-Fuentes, 2018).

Del mismo modo, la reespecificación del modelo se llevó a cabo bajo los criterios de Rial et al. (2006), y este modelo permitió obtener mejores indicadores de ajuste sin afectar la propuesta teórica, permitiendo simultáneamente una primera aproximación a la representación del constructo resiliencia y la obtención de evidencia de validez de constructo. Tras optar por este modelo, se evidencia también que los ítems RFPI21 y RFPI25 están interrelacionados y denotan la importancia que tiene la solución de problemas para favorecer la resiliencia (González-ArratiaLópez-Fuentes, 2018). Si bien el modelo final, con dos errores correlacionados, permite que el modelo alcance un mejor ajuste, es indispensable considerar dos aspectos: 1) se requiere hacer una revisión de los ítems, ya que es posible que los participantes tuvieran cierta dificultad en su comprensión y 2) es conveniente replicar el modelo con diferentes muestras y contrastarlo para verificar su idoneidad.

Respecto a la confiabilidad de la prueba completa, esta es ligeramente menor a lo recomendado por Oviedo y Campo-Arias (2005) y De Vellis (2003). En especial, el Factor 2 (FPE) mostró una confiabilidad baja. Sin embargo, autores como Tristán (2008) sugieren que la confiabilidad puede ser baja de manera inicial, ya que es necesario satisfacer primero criterios de validez y objetividad para después contar con la confiabilidad pertinente. Lo mismo ocurre con el hecho de determinar qué coeficiente de confiabilidad resulta aceptable, sin embargo, se requiere de continuar con una revisión de los ítems para que la confiabilidad sea aceptable, e incluso se incremente, pero sin perder de vista lo que indican Hair et al. (2004, p. 613): “desde la perspectiva práctica como teórica no podemos medir perfectamente un concepto y (...) siempre hay algún grado de error de medida". Así, es imprescindible hacer una reevaluación de los ítems para minimizar el error sistemático, lo cual representa un importante desafío en la medición de la resiliencia.

No obstante, estos resultados ponen de manifiesto la necesidad de continuar con un análisis sobre modelos alternativos, dado que este estudio sólo confirma que este es uno de los varios modelos posibles (Hair et al., 2004). Desde esta línea, se reconoce que el proceso de validación requiere de continuar documentando (Arce-Ferrer \& Corral-Verdugo, 2003).

Si bien esta escala es una medida general de la resiliencia que posee relativa consistencia y validez, es deseable que el instrumento permita la mayor representatividad del constructo bajo estudio. Al mismo tiempo, se debe de tomar en cuenta la naturaleza de la resiliencia, ya que es común 
que las escalas que intentan medirla muestren una aparente inestabilidad, lo cual podría deberse a la variabilidad del constructo resiliencia según el contexto, la edad, el género y el origen cultural (Bonanno, 2004; Mejía, 2003). Sobre todo porque "los estadísticos de fiabilidad varían entre poblaciones y están afectados por otras condiciones como la longitud de la prueba y la variabilidad de las muestras de personas" (Prieto \& Delgado, 2010, p. 70). De ahí que se considere que es indispensable la acumulación de evidencia empírica respecto a su uso con fines de investigación.

Desde el marco ecológico, la medición de la resiliencia requiere de tomar en cuenta la compleja relación individuo-ambiente considerando: 1) la gama amplia de situaciones de crisis o estrés consideradas amenazantes, 2) el momento en que se hizo la medición, si se ha realizado durante el momento de crisis o posterior a la situación, y 3) cuáles son "los indicadores que contribuyan a identificar aquellas instancias en que el retorno a un estado anterior es deseable y aquellas en que no lo es" (Food Security Information Network [FSIN], 2014, p. 10).

Adicionalmente es importante hacer una reflexión sobre la estabilidad de una escala de medida, en la que se debe considerar cuál es el punto de referencia del cual se parte ya que "cada sujeto puede tomar un referente distinto dentro de los que se les presenta y por otra parte cada sujeto le puede dar una puntación inicial distinta" (BlascoLópez \& Martínez-Tercero, 2001, p. 30) de ahí que se recomiende la replicación de la escala en idénticas circunstancias para contar con mayor evidencia empírica.

En el caso del Estudio 2, respecto a la validez de constructo externa, se esperaba que la escala de resiliencia se asociara con las escalas de autoestima y satisfacción con la vida, y efectivamente los resultados indicaron que existe una relación positiva significativa entre las variables.
Esto es coherente con la investigación de Balgiu (2017) y de Gutiérrez y Romero (2014) en el sentido de que la autoestima tiene un efecto positivo sobre la resiliencia e, incluso, sobre la satisfacción con la vida en adolescentes. Lo mismo ocurre con estudios de tipo correlacional, en los que se ha informado que la satisfacción con la vida se asocia con la resiliencia de manera moderada (Casazola-Larota \& Vargas-Mamani, 2018; Guevara-Amasifuen, 2017). Sobre esta línea, los hallazgos aquí obtenidos también coinciden con Bernal-Romero et al. (2015), Pinheiro y Matos (2013) y con Morales-Rodríguez, Díaz-Barajas y Ortíz-Maldonado (2017) quienes, con la misma escala de resiliencia de González-Arratia-LópezFuentes (2018), informaron que los universitarios que cuentan con habilidades resilientes son los que se sienten más satisfechos con su vida. Así, la asociación de la escala de resiliencia con autoestima y satisfacción con la vida, tal como había sido planteada desde la teoría, resulta en evidencia que apoya la validez de constructo del instrumento.

Respecto a las diferencias obtenidas en resiliencia entre niños y niñas, se confirma parcialmente la hipótesis (Abiola \& Udofia, 2011; Sánchez-Teruel y Robles-Bello, 2015) dado que sólo FPE mostró diferencias según el sexo, tal como había sucedido en estudios previos (GonzálezArratia-López-Fuentes \& Valdez-Medina, 2013) en donde también se halló que las niñas mostraron puntajes más altos en este factor, lo que sugiere que ellas tienden a contar con más habilidades interpersonales y fortaleza interna, en tanto que los niños tienden a ser más pragmáticos. Sin embargo, se requiere continuar analizando la variable sexo, ya que aún no se cuenta con suficiente información que explique estos hallazgos.

De manera general, las ventajas de esta versión son que se requiere de un breve tiempo de respuesta y es de fácil aplicación, por lo que resulta ser una opción útil y práctica para indagar 
las características individuales acerca de la capacidad de las personas para enfrentar una situación de crisis y sobreponerse a la adversidad. Estos hallazgos llevan a recomendar su aplicación en su versión breve (14 ítems) en diferentes grupos de edad, así como su uso en muestras hispanohablantes, para posteriormente traducirlo a distintos idiomas con la finalidad de realizar estudios transculturales.

En cuanto a las limitaciones del presente estudio, el hecho de que las muestras no fueron al azar puede ser una importante fuente de sesgo, por lo que se recomienda replicar ambos estudios en una muestra ampliada y diversificada con la finalidad de verificar la consistencia de los datos y su utilidad en diversos contextos. El modelo reespecificado logró índices aceptables, sin embargo, se hace necesario continuar con una validación cruzada, e incluso transformar los datos, lo que significa que no sólo se requiere de utilidad estadística, sino también de utilidad práctica para analizar en mejor medida las relaciones entre las variables y lograr un refinamiento de la medición.

Otro punto importante es que se requiere de mayor investigación para analizar si la escala es una opción para la medición de la resiliencia posterior a un tratamiento, por lo que es indispensable verificar su uso en contextos clínicos. Bajo esta consideración, la escala es un indicador que bien puede complementarse con una entrevista para obtener mayor información respecto a la capacidad resiliente de los individuos. En virtud de que se trata de un estudio transversal, se recomienda dar seguimiento a los individuos, por lo que el estudio longitudinal sería la estrategia fundamental para conocer aún más sobre el modo de recuperación de los niños frente a la precariedad económica.

Así y sin perder de vista la limitación sobre la representatividad de la prueba, estos hallazgos aportan evidencias de su validez, así como de su utilidad en contextos educativos con fines de investigación, a los fines de evaluar procesos o el efecto de programas de intervención en niños en situación de vulnerabilidad.

Finalmente, hay que tener presente que la complejidad del constructo resiliencia se debe a que es multidimensional, por lo que es necesario incluir otros indicadores. Por este motivo se plantea para futuros estudios la necesidad de que en la medición de la resiliencia también se analicen la situación de crisis inicial, así como las diferentes formas de recuperación y su aplicación en muestras clínicas, pues se requiere aún detectar los posibles cambios tras una intervención.

\section{Conclusiones}

Con base en los resultados obtenidos, se considera que la escala de resiliencia GA-RE14 en su versión breve tiene adecuadas propiedades psicométricas para su uso en escolares. Así, esta versión puede ser utilizada para la medición de características resilientes desde la perspectiva de Henderson-Grotberg (2006) y representa una opción práctica para la medición de la resiliencia en niños en una situación de vulnerabilidad psicosocial como lo es la pobreza. Sus propiedades psicométricas resultaron ser en general, satisfactorias, sin embargo, es indispensable continuar con análisis rigurosos sobre su uso en otros contextos.

\section{Referencias}

Abad, F., Garrido, J., Olea, J., \& Ponsoda, V. (2006). Introducción a la Psicometría. Teoría Clásica de los Tests y Teoría de la Respuesta al Ítem. Madrid, España: Universidad Autónoma de Madrid.

Abad, F., Olea, J., Ponsoda, V., \& García, C. (2011). Medición en Ciencias Sociales y de la Salud. Madrid, 
España: Síntesis.

Abiola, T., \& Udofia, O. (2011). Psychometric assessment of the Wagnild and Young's resilience scale in Kano, Nigeria. BMC Research Notes, 4(1), 509. doi: 10.1186/1756-0500-4-509

Arbuckle, J. L. (2011). Amos (Version 20.0). Chicago: IBM SPSS.

Arce-Ferrer, A. J., \& Corral-Verdugo, V. (2003). La medición de la aptitud académica general: Estudio de un caso en el ingreso a la licenciatura. En D. González (Ed.), Modelamiento Estructural en las Ciencias Sociales (pp. 85-109). México: Universidad de Sonora.

Asociación Americana de Psicología. (2010). Manual de Publicaciones de la American Psychological Association. D. F., México: Manual Moderno.

Balgiu, B. A. (2017). Self-esteem, personality and resilience. Study of a students emerging adults group. Journal of Educational Sciences \& Psychology, 7(69), 93-99. Recuperado de http://jesp.upg-ploiesti. ro

Becoña-Iglesias, E. (2006). Resiliencia: Definición, características y utilidad del concepto. Revista de Psicopatología y Psicología Clínica, 11(3), 125-146. doi: 10.5944/rppc.vol.11.num.3.2006.4024

Benetti, C., \& Kambouropoulos, N. (2006). Affect-regulated indirect effects of trait anxiety and trait resilience on self-esteem. Personality and Individual Differences, 41(2), 341-352. doi: 10.1016/j.paid.2006.01.015

Bernal-Romero, T., Daza-Pinzón, C., \& Jaramillo-Acosta, P. (2015). Satisfacción con la vida y resiliencia en jóvenes en extraedad escolar. Revista Iberoamericana de Psicología: Ciencia y Tecnología, 8(2), 43-53. Recuperado de https://reviberopsicologia.ibero.edu. co/index

Blasco-López, M. F., \& Martínez-Tercero, M. (2001). La estabilidad de las escalas psicológicas de intervalo: Una aplicación sobre la medida de la actitud. Esic Market, 110, 24-42.

Bollen, K. A., \& Stine, R. (1990). Direct and indirect effects: Classical and bootstrap estimates of variabil- ity. Sociological Methodology, 20, 115-140. doi: $10.2307 / 271084$

Bonanno, G. A. (2004). Loss, trauma and human resilience: Have we underestimated the human capacity to thrive after extremely aversive events? American Psychologist, 59(1), 20-28. doi: 10.1037/0003-066X.59.1.20

Bronfenbrenner, U. (1979). The ecology of human development: Experiments by nature and design. Cambridge, MA: Harvard University.

Byrne, M. B. (2010). Structural Equation Modeling with AMOS. Basic concepts, applications and programming ( $2^{\mathrm{a}}$ ed.). New York, NY: Routledge. doi: $10.4324 / 9780203805534$

Campbell-Sills, L., \& Stein, M. B. (2007). Psychometric analysis and refinement of the Connor-Davidson Resilience Scale (CD-RISC): Validation of a 10Item measure of resilience. Journal of Traumatic Stress, 20(6), 1019-1028. doi: 10.1002/jts.20271

Casazola-Larota, B., \& Vargas-Mamani, L. M. (2018). Relación entre resiliencia y satisfacción con la vida en adolescentes de 15 a 18 años del Distrito de Sachaca. (Tesis de grado). Facultad de Psicología. Universidad Nacional de San Agustín de Arequipa, Perú. Recuperado de http://repositorio.unsa.edu.pe/ handle/UNSA/6606

Castro, C. V. A. (2016). Variables Psicológicas (resiliencia, optimismo y estrategias de afrontamiento) que inciden en el Bienestar Psicológico de pacientes diagnosticados con DM2. (Disertación doctoral inédita). Facultad de Enfermería y Obstetricia. Universidad Autónoma del Estado de México.

Connor, K. M., \& Davidson, J. R. T. (2003). Development of a new resilience scale: The Connor-Davidson Resilience Scale (CD-RISC). Depression and Anxiety, 18(2), 76-82. doi: 10.1002/da.10113

Consejo Nacional de Evaluación de la Política de Desarrollo Social. (2008-2018). Informe Anual Sobre la Situación de Pobreza y Rezago Social. México: Consejo Nacional de Evaluación de la Política de Desarrollo Social. Recuperado de https://www.gob. 
mx/cms/uploads/attachment/file/42741/Mexico_106. pdf

Consejo Nacional de Evaluación de la Política de Desarrollo Social. (2013). Informe de pobreza y evaluación Estado de México 2012-2013. Recuperado de https://www.coneval.org.mx/coordinacion/entidades/ Documents/Informes\%20de\%20pobreza\%20y\%20 evaluaci\%C3\%B3n\%202012-2013_Documentos/ IPE\%20MEXICO.pdf

De Vellis, R. F. (2003). Scale Development. Theory and applications ( $2^{\mathrm{a}}$ ed.). Thousand Oaks, CA: Sage.

Díaz, C. R. (2019). Espiritualidad como factor de protección para la resiliencia ante situaciones estresantes en adultos mayores. (Disertación doctoral inédita). Facultad de Enfermería y Obstetricia. Universidad Autónoma del Estado de México.

Diener, E., Emmons, R., Larsen, R. J., \& Griffin, S. (1985). The Satisfaction With Life Scale. Journal of Personality Assessment, 49(1), 71-75. doi: 10.1207/ s15327752jpa4901_13

Ferrando, P. J., \& Anguiano-Carrasco, C. (2010). El análisis factorial como técnica de investigación en psicología. Papeles del Psicólogo, 31(1), 18-33. Recuperado de http://www.papelesdelpsicologo.es/home

Food Security Information Network. (2014). Principios sobre la medición de la resiliencia. Serie Técnica 1. Recuperado de http://www.fsincop.net/topics/es

Fouladi, R. T. (Abril, 1998). Covariance structure analysis techniques under conditions of multivariate normality and nonnormality-modified and bootstrap test statistics. Trabajo presentado en la American Educational Research Association Annual Meeting. San Diego, CA. https://files.eric.ed.gov/fulltext/ ED420717.pdf

González-Álvarez, N., Abad-González, J. I., \& LévyMangin, J. P. (2006). Normalidad y otros supuestos en análisis de covarianzas. En J. P. Lévy-Mangin, \& J. Varela-Mallou (Eds.). Modelización con estructuras de covarianzas en Ciencias Sociales. Temas esenciales, avanzados y aportaciones especiales (pp. 31-57). España: Netbiblo.
González-Arratia-López-Fuentes, N. I. (2018). Autoestima, optimismo y resiliencia en niños en situación de pobreza. Revista Internacional de Psicología, 16(1), 1-119. doi: 10.33670/18181023.v16i01.261

González-Arratia-López-Fuentes, N. I., Morelato, G., González-Escobar, S., \& Ruíz-Martínez, A. O. (2018). Factores de riesgo y protección para la resiliencia en niños mexicanos y argentinos. En R. DíazLoving, I. Reyes-Lagunes \& R. F. López (Eds.), Aportaciones actuales de la Psicología Social. Vol IV, (pp. 2523-2539). México: Asociación Mexicana de Psicología Social.

González-Arratia-López-Fuentes, N. I., \& Valdez-Medina, J. L. (2012). Análisis factorial confirmatorio del Cuestionario de Resiliencia en una muestra de niños. En R. Díaz-Loving, S. Rivera \& I. Reyes-Lagunes (Eds.), La Psicología Social en México. Vol. XIV, (pp. 676-681). México: Asociación Mexicana de Psicología Social.

González-Arratia-López-Fuentes, N. I., \& Valdez-Medina, J. L. (2013). Resiliencia: Diferencias por edad en hombres y mujeres mexicanos. Acta de Investigación Psicológica, 3(1), 941-955. doi: 10.1016/ s2007-4719(13)70944-x

Gosling, S. D., Rentfrow, P. J., \& Swann Jr., W. B. (2003). A very brief measure of the Big-Five personality domains. Journal of Research in Personality, 37(6), 504-528. doi: 10.1016/S0092-6566(03)00046-1

Guevara-Amasifuen, S. (2017). Resiliencia y satisfacción con la vida en estudiantes de la Universidad Nacional de Educación "Enrique Guzmán y Valle". (Disertación de maestría) Universidad Nacional de Educación Enrique Guzmán y Valle, Lima, Perú. Recuperado de http://repositorio.une.edu.pe

Gutiérrez, M., \& Romero, I. (2014). Resiliencia, bienestar subjetivo y actitudes de los adolescentes hacia el consumo de drogas en Angola. Anales de Psicología, 30(2), 608-619. doi: 10.6018/analesps.30.2.148131

Hair, J., Anderson, R., Tatham, R., \& Black, W. (2004). Análisis Multivariante ( $5^{\mathrm{a}}$ ed.). México: Prentice-Hall. 
Hancock, G. R., \& Nevitt, J. (1999). Bootstrappring and the identification of exogenous latent variables within structural equation models. Structural Equation Modeling: A Multidisciplinary Journal, 6(4), 394399. doi: 10.1080/10705519909540142

Henderson-Grotberg, E. (2006). La resiliencia en el mundo de hoy. Cómo superar las adversidades. Barcelona, España: Gedisa.

Hernández-Contreras, C., \& Rivera-Ottenberger, O. D. (2018). Adaptación transcultural y evaluación de las estructuras factoriales del Test de Adicción a Internet en Chile: Desarrollo de una versión abreviada. Revista Iberoamericana de Diagnóstico y Evaluación, 49(4), 143-155. doi: 10.21865/RIDEP49.4.12

Hu, L. T., \& Bentler, P. M. (1998). Fit indices in covariance structure modeling: Sensitivity to underparameterized model misspecification. Psychological Methods, 3(4), 424-453. doi: 10.1037//1082-989x.3.4.424

Hurtes, K. P., \& Allen, L. R. (2001). Measuring resiliency in youth: The resiliency attitudes and skills profile. Therapeutic Recreation Journal, 35(4), 333-347. Recuperado de https://js.sagamorepub.com/trj

IBM Corporation. (2011). IBM SPSS Statistics 20.0. [software de cómputo]. Armonk, NY: IBM.

Jew, C. L., Green, K. E., \& Kroger, J. (1999). Development and validation of a measure of resiliency. Measurement and Evaluation in Counseling and Development, 32(2), 75-89. doi: 10.1080/07481756.1999.12068973

Jowkar, B., Friborg, O., \& Hjemdal, O. (2010). Cross-cultural validation of the Resilience Scale for Adults (RSA) in Iran. Scandinavian Journal of Psychology, 51(5), 418-425. doi: 10.1111/j.1467-9450.2009.00794.x

Kalawski, J. P., \& Haz, A. M. (2003). Y... ¿dónde está la resiliencia? Una reflexión conceptual. Interamerican Journal of Psychology, 37(2), 365-372. Recuperado de https://journal.sipsych.org/index.php/IJP

Kenneally-Nicholas, P. (1993). Hardiness, self-care practices and perceived health status in older adults. Journal of Advances Nursing, 18(7), 1085-1094. doi: 10.1046/j.1365-2648.1993.18071085.x

Lévy-Mangin, J. P., Martín-Fuentes, M. T., \& Román-
González, M. V. (2006). Optimización según estructuras de covarianzas. En J. P. Lévy-Mangin \& J. Varela-Mallou (Eds.), Modelización con estructuras de covarianzas en Ciencias Sociales (pp. 11-30). España: Netbiblo.

Lévy-Mangin, J. P., \& Varela-Mallou, J. (2006). Modelización con estructuras de covarianzas en Ciencias Sociales. España: Netbiblo. doi: 10.4272/84-9745-136-8

Lloret-Segura, S., Ferreres-Traver, A., Hernández-Baeza, A., \& Tomás-Marco, I. (2014). El análisis factorial exploratorio de los ítems: Una guía práctica, revisada y actualizada. Anales de Psicología, 30(3), 11511169. doi: 10.6018/analesps.30.3.199361

Loo, R., \& Thorpe, K. (2000). Confirmatory factor analyses of the full and short versions of the MarloweCrowne Social Desirability Scale. The Journal of Social Psychology, 140(5), 628-635. doi: 10.1080/00224540009600503

López-Fuentes, I., \& Calvete, E. (2016). Desarrollo del inventario de factores de resiliencia ante la adversidad. Ansiedad y Estrés, 22(2-3), 110-117. doi: 10.1016/j. anyes.2016.10.003

Luthar, S. S., \& Bidwell-Zelazo, L. (2007). Research on resilience. An integrative review. En S. Luthar (Ed.), Resilience and vulnerability. Adaptation in the context of childhood adversities (pp. 510-549). Estados Unidos: University Cambridge. doi: 10.1017/ cbo9780511615788.023

Masten, A. S. (2007). Resilience in developing systems: Progress and promise as the fourth wave rises. Development and Psychopathology, 19(3), 921-930. doi: 10.1017/S0954579407000442

Mejía, R. (2003). Resiliencia: Ilusión o realidad? Revista CES Medicina, 17(1), 57-62. Recuperado de http:// revistas.ces.edu.co/index.php/medicina/index

Mondragón-Barrera, M. A. (2014). Uso de la correlación de Spearman en un estudio de intervención en fisioterapia. Movimiento Científico, 8(1), 98-104. doi: 10.33881/2011-7191.mct.08111

Montero, I., \& León, O. G. (2002). Clasificación y des- 
cripción de las metodologías de investigación en Psicología. Revista Internacional de Psicología Clínica y Salud, 2(3), 503-508. Recuperado de https://www.redalyc.org

Montero-Rojas, E. (2013). Referentes conceptuales y metodológicos sobre la noción moderna de validez de instrumentos de medición: Implicaciones para el caso de personas con necesidades educativas especiales. Actualidades en Psicología, 27(114), 113-128. doi: 10.15517/ap.v27i114.7900

Morales-Rodríguez, M., Díaz-Barajas, D., \& OrtízMaldonado, D. (2017). Autoeficacia, resiliencia y satisfacción con la vida en universitarios de escuelas públicas y privadas: Abordaje desde el modelo de desarrollo positivo. Memoria in extenso presentada en el XIV Congreso Nacional de Investigación Educativa, COMIE. San Luis, Potosí. Recuperado de http://www.comie.org.mx/v5/sitio

Moskovitz, S. (1983). Love despite hate. Child survivors of the Holocaust and their adult lives. New York, NY: Schocken.

Ordaz-Baz, M. B. (2008). Introducción a la Metodología SEM: Concepto y propósitos fundamentales. En M. A. Verdugo, M. Crespo, M. Badía \& B. Arias (Coords.), Metodología en la Investigación sobre discapacidad. Introducción al uso de las ecuaciones estructurales. VI Seminario Cientifico, SAID (pp. 1328). Salamanca, España: INICO . Recuperado de https://sid.usal.es

Oshio, A., Kaneko, H., Nagamine, S., \& Nakaya, M. (2003). Construct validity of the Adolescent Resilience Scale. Psychological Reports, 93(3), 1217-1222. doi: 10.2466/pr0.2003.93.3f.1217

Ospina-Muñoz, D. E. (2007). La medición de la resiliencia. Investigación y Educación en Enfermería, 25(1), 5865. Recuperado de https://aprendeenlinea.udea.edu. co/revistas/index.php/iee/index

Oviedo, H. C., \& Campo-Arias, A. (2005). Aproximación al uso del coeficiente alfa de Cronbach. Revista Colombiana de Psiquiatría, 34(4), 572-580. Recuperado de https://website.psiquiatria.org.co
Padrós-Blázquez, F., Gutiérrez-Hernández, C. Y., \& Medina-Calvillo, M. A. (2015). Propiedades psicométricas de la Escala de Satisfacción con la Vida (SWLS) de Diener en población de Michoacán (México). Avances en Psicología Latinoamericana, 33(2), 223-232. doi: 10.12804/ap133.02.2015.04

Palomar-Lever, J. (2015). Resiliencia, educación y movilidad social en adultos beneficiarios del Programa de Desarrollo Humano Oportunidades. D. F., México: Universidad Iberoamericana.

Perpiñá-Galvañ, J., Richart-Martínez, M., CabañeroMartínez, M. J., \& Martínez-Durá, I. (2011). Validez de contenido de versión corta de la subescala del Cuestionario State-Trait Anxiety Inventory (STAI). Revista Latino-Americana de Enfermagem, 19(4), 1-6. doi: 10.1590/s0104-11692011000400005

Pinheiro, M. R., \& Matos, A. P. (2013). Exploring the construct validity of the two versions of the Resilience Scale in an Portuguese adolescent sample. The European Journal of Social \& Behavioural Sciences, 2(2), 178-189. doi: 10.15405/futureacademy/ ejsbs(2301-2218).2012.2.5

Plata, Z. L. D. (2013). Resiliencia, autoestima y autoeficacia como predictores del rendimiento escolar en educación básica. (Disertación de maestría inédita). Facultad de Ciencias de la Conducta. Universidad Autónoma del Estado de México.

Pourtois, J. P. (2014). Los recursos de la resiliencia. En J. M. Madariaga (Coord.), Nuevas miradas sobre la resiliencia. Ampliando ámbitos y prácticas (pp. 69110). Barcelona, España: Gedisa.

Prado-Álvarez, R., \& del Águila-Chávez, M. (2003). Diferencia en la resiliencia según género y nivel socioeconómico en adolescentes. Persona, 6, 179-196. doi: 10.26439/persona2003.n006.885

Prieto, G., \& Delgado, A. R. (2010). Fiabilidad y Validez. Papeles del Psicólogo, 31(1), 67-74. Recuperado de http://www.papelesdelpsicologo.es/home

Rial, A., Varela-Mallou, J., Abalo, J., \& Lévy-Mangin, J. P. (2006). El análisis factorial confirmatorio. En J. P. Lévy-Mangin, \& J. Varela-Mallou (Eds.), 
Modelización con estructuras de covarianzas en Ciencias Sociales (pp. 119-154). España: Netbiblo.

Rocha-Romero, L. A. (2017). Ansiedad, depresión y resiliencia en personas con IRC. (Disertación). Facultad de Ciencias de la Conducta. Universidad Autónoma del Estado de México. Recuperado de http://ri.uaemex.mx

Rodríguez-Naranjo, C., \& Caño-González, A. (2012). Autoestima en la adolescencia: Análisis y estrategias de intervención. International Journal of Psychology and Psychological Therapy, 12(3), 389403. Recuperado de https://www.ijpsy.com

Ruíz-González, P., Medina-Mesa, Y., Zayas, A., \& GómezMolinero, R. (2018). Relación entre la autoestima y la satisfacción con la vida en una muestra de estudiantes universitarios. International Journal of Developmental and Educational Psychology. Revista INFAD de Psicología, 2(1), 67-76. doi: 10.17060/ijodaep.2018.n1.v2.1170

Rutter, M. (1999). Resilience concepts and findings: Implications for family therapy. Journal of Family Therapy, 21(2), 119-144. doi: 10.1111/1467-6427.00108

Salgado-Levano, A. C. (2005). Métodos e instrumentos para medir la resiliencia: Una alternativa peruana. Liberabit. Revista Peruana de Psicología, 11(11), 41-48. Recuperado de http://revistaliberabit.com/es

Sánchez-Teruel, D., \& Robles-Bello, M. A. (2015). Escala de Resiliencia 14 ítems (RS-14): Propiedades psicométricas de la versión en español. Revista Iberoamericana de Diagnóstico y Evaluación, 2(40), 103-113. Recuperado de https://www.aidep.org/es

Sârbescu, P., Rusu, S., \& Costea, I. (2012). The MarloweCrowne Social Desirability Scale in Romania: The development of a 13-item short form. Romanian Journal of Experimental Applied Psychology, 3(2), 37-44. Recuperado de http://www.rjeap.ro

Serrano-Parra, M. D., Garrido-Abejar, M., Notario-Pacheco, B., Bartolomé-Gutierrez, R., Solera-Martínez, M., \& Martínez-Vizcaino, V. (2012). Validez de la escala de Resiliencia de Connor-Davidson (CD-RISC) en una población de mayores entre 60 y 75 años. International Journal of Psychological Research, 5(2), 49-57. doi: 10.21500/20112084.736

Shin, G. S., Choi, K. S., Jeong, K. S., Min, Y. S., Ahn, Y. S., $\&$ Kim, M. G. (2018). Psychometric properties of the 10-ítem Conner-Davinson Resilience Scale on toxic chemical-exposed workers in South Korea. Annals of Occupational Environmental Medicine, 30(1), 5259. doi: 10.1186/s40557-018-0265-5

Stratta, P., Capanna, C., Patriarca, S., de Cataldo, S., Bonanni, R. L., Riccardi, I. \& Rossi, A. (2013). Resilience in adolescence: Gender differences two years after the earthquake of L'Aquila. Personality and Individual Differences, 54(3), 327-331. doi: 10.1016/j.paid.2012.09.016

Toribio-Perez, L. (2017). Factores protectores de la salud mental positiva en adolescentes. (Disertación doctoral). Facultad de Enfermería y Obstetricia. Universidad Autónoma del Estado de México. Recuperado de http://ri.uaemex.mx

Torreblanca-Murillo, L. (2017). Desarrollo de un nuevo instrumento de evaluación: El Cuestionario Breve de Personalidad (CBP). (Disertación doctoral). Facultad de Psicología. Universidad de Málaga, España. Recuperada de https://riuma.uma.es/xmlui

Tristán, L. A. (2008). Elementos de diseño y análisis de instrumentos objetivos. Bogotá, Colombia: Universidad Nacional de Colombia. Instituto de Evaluación e Ingeniería Avanzada.

Verdugo, M. A., Crespo, M., Badía, M., \& Arias, B. (2008). Metodología en la investigación sobre discapacidad. Introducción al uso de las ecuaciones estructurales. VI Seminario Cientifico, SAID. Salamanca, España: INICO.

Wagnild, G. M., \& Young, H. M. (1993). Development and psychometric evaluation of the Resilience Scale. Journal of Nursing Measurement, 1(2), 165-178. 